\title{
Associations of Y-chromosome subdeletion gr/gr with the prevalence of Y-chromosome haplogroups in infertile patients
}

\author{
Mohammad Shahid ${ }^{1,2}$, Varinderpal S Dhillon ${ }^{\star, 3}$, Hesham Saleh Khalii ${ }^{4}$, Anubha Sexana ${ }^{2,5}$ \\ and Syed Akhtar Husain ${ }^{2}$
}

\begin{abstract}
Microdeletions in azoospermia factor (AZF) region on distal $\mathrm{Yq}$ are associated with male infertility and spermatogenic failure due to intra-chromosomal homologous recombination between large nearly identical repeat amplicons and are found in $\sim 10 \%$ of azoospermic and severe oligozoospermic cases. Although AZFc is deleted in azoospermia or oligozoospermia, no definitive conclusion has been drawn for the role of partial AZFC deletions to spermatogenic failure. Therefore, this study is planned to investigate the role of $\mathrm{gr} / \mathrm{gr}$ subdeletions in individuals with spermatogenic failure and to find its relationship with $\mathrm{Y}$ chromosome haplogroups (HGs) in infertile men from Indian population. It is a case-control study involving 236 azoospermic, 182 oligospermic and 240 healthy normozoospermic men. We found $18 \mathrm{gr} / \mathrm{gr}, 11 \mathrm{~b} 1 / \mathrm{b} 3$ and $2 \mathrm{~b} 2 / \mathrm{b} 3$ subdeletions in azoospermic patients and $12 \mathrm{gr} / \mathrm{gr}, 5 \mathrm{~b} 1 / \mathrm{b} 3$ and $4 \mathrm{~b} 2 / \mathrm{b} 3$ subdeletions in oligospermic patients. However, we also found seven gr/gr deletions in normozoospermic men. Seven patients each with spermatogenic arrest and oligospermia who carry gr/gr subdeletions have deleted DAZ3/DAZ4 genes. A total of 11 patients with sertoli cell-only syndrome (SCOS) and 5 oligospermic patients with gr/gr subdeletions also have $D A Z 1 / D A Z 2$ genes deleted indicating that deletions of $D A Z$ genes contributed differently to damage to spermatogenic process. L1 HG is found in patients showing b1/b3 subdeletions, whereas $\mathrm{HG} \mathrm{H1a2}$ and $\mathrm{H} 1 \mathrm{~b}$ were found in normozoospermic individuals with gr/gr subdeletions. Our results provide evidence of association between the occurrence of subdeletions and male infertility as well as the severity of the spermatogenic failure.
\end{abstract}

European Journal of Human Genetics (2011) 19, 23-29; doi:10.1038/ejhg.2010.151; published online 8 September 2010

Keywords: spermatogenic arrest; sertoli cell-only syndrome; oligospermia; AZFc subdeletions; haplogroup

\section{INTRODUCTION}

Infertility affects $10-15 \%$ of the human couples and spermatogenic failure is among the main reason in $50 \%$ of these cases. ${ }^{1}$ Male-specific region of the $\mathrm{Y}$ chromosome has an important role in sex determination and spermatogenesis. ${ }^{2}$ This region consists of long, Y-specific repeat sequences termed as amplicons. Many amplicons contain genes that have testes-specific patterns and perform critical functions. ${ }^{1}$ Homologous recombination between these repeats can lead to deletions which cause spermatogenic failure. ${ }^{3}$ Majority of these deletions fall within the azoopsermia factor (AZF) region which is further divided as: $\mathrm{AZFa}, \mathrm{AZFb}$ and $\mathrm{AZFc}{ }^{4}$ Deletions in AZFa region usually lead to sertoli cell-only syndrome (SCOS), complete deletion(s) of either $\mathrm{AZFb}$ or $\mathrm{AZFb}$ and $\mathrm{AZFc}$ lead to azoospermia associated with SCOS or pre-meiotic spermatogenic arrest (SA). 2,5,6 The azoospermia factor $\mathrm{c}(\mathrm{AZFc})$ region is highly variable because of many recurrent intra-chromosomal homologous recombination ${ }^{7,8}$ and its deletion may influence susceptibility to spermatogenic failure. ${ }^{1,9}$ The complete AZFc (b2/b4) deletion, spaning approximately $3.5 \mathrm{Mb}$ removes eight gene families including all deleted in azoospermia $(D A Z)$ gene family members that represent the strongest candidate responsible for the AZFc phenotype linked with spermatogenic failure. ${ }^{7,10,11}$ However, the recently described $1.6 \mathrm{Mb}$ deletion polymorphism turned out to be the most frequent large Y chromosome deletion. ${ }^{12}$ This deletion (gr/gr) affects AZFc region of $Y$ chromosome and also removes two copies of $D A Z$ gene and several other transcriptional units. A few studies have presented the evidence that low penetrance gr/gr deletions may lead to spermatogenic failure. ${ }^{12,13-17}$ However, others reports failed to show any phenotypic impact of gr/gr deletions on spermatogenic process. ${ }^{18-21}$ In the past decade, studies have suggested predisposition of genetic background to spermatogenic failure in Japanese, Danish and Chinese populations. ${ }^{22-24}$ It has been shown that DAZ1/DAZ2 deletions are restricted to gr/gr carriers with declining sperm quantity. ${ }^{14,25,26}$ Recent advances in this field has further helped the researchers to better define 'gr/gr deletions' as 'gr/gr deletion rearrangements' with respect to the gene dosage and copy number analysis of chromodomain $\mathrm{Y} 1(C D Y 1)$ and $D A Z .^{27}$

Above findings suggest that $\mathrm{Y}$ chromosome is highly variable and these variations may generate a genetic background for the susceptibility to Y-related spermatogenic failure. Therefore, this study is designed to ascertain (i) the frequency of subdeletions and verify their association with fertility and (ii) the possible association of Y-chromosome background with the failure of the spermatogenic

${ }^{1}$ College of Dentistry, Alkharj University, Alkharj, Kingdom of Saudi Arabia; ${ }^{2}$ Human Genetics Laboratory, Department of Biosciences, Jamia Millia Islamia (a Central University), New Delhi, India; ${ }^{3}$ Genome Stability Laboratory, CSIRO Food and Nutritional Sciences, Adelaide, Australia; ${ }^{4}$ Department of Oral and Maxillofacial Surgery, College of Dentistry, King Saud University, Riyadh, Kingdom of Saudi Arabia; ${ }^{5}$ Brain and Mind Research Institute, University of Sydney, Sydney, NSW, Australia

${ }^{*}$ Correspondence: Dr VS Dhillon, Research Scientist, CSIRO Food and Nutritional Sciences, Gate 13, Kintore Avenue, PO BOX 10041 , Adelaide BC, Adelaide 5000, Australia. Tel: +61 88303 8932; Fax: +61 88303 8899; E-mail: varinderpal.dhillon@csiro.au

Received 26 November 2009; revised 24 June 2010; accepted 30 July 2010; published online 8 September 2010 
process. To the best of our knowledge, this is the first report from India that links gr/gr and other subdeletions with infertility.

\section{MATERIALS AND METHODS}

\section{Patients}

The study was approved by the Hospital and University Ethics Committee and informed consent was obtained from each subject. A total of 465 idiopathic infertile men were referred to our laboratory for genetic investigations. Of these 27 were found to have either numerical or structural chromosome abnormalities and hence were omitted from this study. We found 20 patients with complete AZFc deletions. As the main focus of this study is on the frequency of subdeletions, therefore, these 20 patients with complete deletions were also omitted from this study. Hence, this study comprises of 418 infertile men (236 azoospermic and 182 oligospermic). In all, 240 fertile men who are normozoospermic and have fathered at least one child were selected as control group. Infertile men were recruited from the Male Infertility Clinic (Department of Family Welfare Centre), Lok Nayak Jai Prakash and Associated Hospitals, New Delhi, India. In case with azoospermic men, testicular cytology was performed with the use of fine needle aspiration cytology as per patients' consent. SCOS was diagnosed when all testicular material obtained from both testes contained only sertoli cells without any germ cells. SA was diagnosed when tubules contained no spermatozoa except developing spermatocytes and/or spermatids. Using above-mentioned criteria, we selected patients with azoospermia who either had bilateral SCOS or SA with normal karyotype. On the basis of semen analysis and WHO criteria, ${ }^{28}$ idiopathic infertile patients were divided into two groups: non-obstructive azoospermic (no sperm in ejaculate even after centrifugation) and oligospermic (sperm concentration $0.1-20 \times 10^{6} / \mathrm{ml}$ ). The exclusion criteria include excessive alcohol consumption, smoking, use of drugs that include ecstasy, marijuana and recreational substances, exposure to radiations as part of radiotherapy. The patients were also checked for the history of relevant medical disorders, for example, mumps, diabetes, liver and renal diseases, endocrine abnormality (hypogonadism), exposure to environmental toxins (including pesticides and lead) and/or medication affecting spermatogenesis, gross dysmorphic abnormalities, acquired and congenital structural defects of urogenital system (cystic fibrosis, Young's syndrome, etc) and history of surgical intervention of genital tract obstruction/dysfunction. The patients showing any of these symptoms were excluded from this study. All members of the normal control group included as healthy men with normal reproductive history and normal physical examination. These were matched with respect to age, caste (ethnic) and region to prevent the confounding of the results because of the population stratification.

\section{Molecular analysis}

Genomic DNA was prepared from peripheral blood samples using standard techniques. Analysis of AZFc-specific STSs was performed on genomic DNA by PCR using sY1291 (specific for the gr/gr-g1/g2 and b1/b3 deletions) and sY1191 (specific for the b2/b3 and rg/gr-g1/g3 deletions). Analysis was further confirmed with additional STSs: sY142, sY1258, sY1161, sY1197, sY1206 and sY1201. ${ }^{12}$ Two multiplex reactions were performed, one with primers sY1291, sY1191 and sY1161, and the other with sY1201 and sY1206 where as other markers were used in single primer pair PCR. PCR with single primer pair was used to confirm the deletions. All PCR assays were performed in a total volume of $25 \mu \mathrm{l}$ containing $50 \mathrm{ng}$ of each DNA sample, $1 \times$ PCR buffer, $1.5 \mathrm{mmol} / / \mathrm{MgCl}_{2}, 200 \mu \mathrm{mol} / \mathrm{l} \mathrm{dNTP}$, 5 pmol of each primer pair and 1 IU Taq DNA polymerase (Perkin-Elmer Cetus, Norwalk, CT, USA). The cycling protocol was as follows: 5 min at $94^{\circ} \mathrm{C}$, followed by 35 cycles as $94{ }^{\circ} \mathrm{C}$ for $45 \mathrm{~s}, 55-62{ }^{\circ} \mathrm{C}$ for $45-60 \mathrm{~s}$ and $60 \mathrm{~s}$ at $72^{\circ} \mathrm{C}$, and $72{ }^{\circ} \mathrm{C}$ for $5 \mathrm{~min}$. The PCR products were analysed by electrophoresis at $100 \mathrm{~V}$ on $2 \%$ agarose gels (Sigma, St Louis, MO, USA). Suitable positive and negative controls were included in every PCR. In addition to these, we also analyzed the $D A Z$ copy type by single nucleotide variant (SNV) method as described previously. ${ }^{29}$ Briefly, analyses of DAZ-specific SNVs for determining DAZ gene copy types was carried out for three SNVs (sY581, sY587 and SNVII) by PCR amplification-restriction digestion assay and the mutations confirmed by direct sequencing. sY581-Sau3A distinguishes DAZ2/DAZ3 ( $\mathrm{T}$ at position 125) from DAZ1/DAZ4 (C at position 125), sY587-Dral distinguishes DAZ1/DAZ2 (T at position 146) from DAZ3/DAZ4 (C at position 146), and SNVII-MboI distinguishes DAZ1 (G at position 121) from DAZ2/DAZ3/DAZ4 (A at position 121). Previously published method was used to study the qualitative and quantitative analysis of copy number of $D A Z$ and $C D Y 1 a / C D Y 1 b$ genes. ${ }^{18}$ Briefly, the $D A Z$ dosage method refer to the simultaneous amplification of a fragment within intron 10 from $A Z F C D A Z$ copies and from its homolog DAZL (localized outside the $A Z F c$ interval), using a single primer pairs o1130 and o1313. This intron is present in just one copy per $D A Z$ or $D A Z L$ gene (as per $A Z F c$ reference structure in normal $46, \mathrm{XY}$ man, there are four DAZ copies and two DAZL copies). Thus, DAZL acts as an internal standard with a known number of copies. The primers flank an insertion/deletion difference of $3 \mathrm{bp}$, which allowed two PCR products generated as $214 \mathrm{bp}$ for $D A Z$ and $217 \mathrm{bp}$ for DAZL. Primer (o1130) was labeled at its $5^{\prime}$ end with FAM. The PCR product was mixed with formamide, denatured at $95^{\circ} \mathrm{C}$ for $5 \mathrm{~min}$ and the different sized loci separated on an automatic sequencer (ABI PRISM 310 Genetic Analyzer PE, Foster city, CA, USA). Quantification was performed by comparing the peak area corresponding to the DAZ locus and to its homolog DAZL. Using the similar approach, we performed the quantitative analysis for $C D Y 1$ copies. In the reference sequence of the $A Z F c$ region, there are two copies of both $C D Y 1$ and $C D Y 2$, which share 98\% nucleotide identity. PCR was set us to amplify CDY1 and CDY2 copies to give fragments of $134 \mathrm{bp}$ for $C D Y 1$ and $137 \mathrm{bp}$ for CDY2 (primers: oMY953a/ o1023). Primer oMY953a was labeled at its $5^{\prime}$ end FAM. Quantification was performed using the same approach as described above - comparing the peak area corresponding to the CDY1 locus and to its homolog CDY2.

\section{Y-haplogroup (HG) analysis}

The Y-chromosome haplotype analyses were performed hierarchically as proposed previously. ${ }^{30,31}$ The UEPs used were as follows: M294, RPS4Y, M216, M089, M213, M069, M052, M089, M197, M097, M039, M370, P80, P266, 12f2.1, M304, M267, M410, M172 M241, M009, M020, M076, M214, M175, M268, M095, M122, M134, M045, M207, M173, M017 and M124. All UEPs (except 12f2.1) were analysed using a mini-sequencing protocol as described elsewhere. ${ }^{32}$

\section{Statistical analysis}

Differences among frequencies were calculated with both $\chi^{2}$-test and Fisher's exact test. Probability $(P)$ values $<0.05$ were regarded as statistically significant. Continuous variables were analysed by the $t$-test for independent samples. Odds ratios (ORs) were also calculated to find out the risk of getting the condition.

\section{RESULTS}

This comprises of 418 infertile men. Of these, 181 were classified with SCOS, 55 with SA and 182 with oligospermia. We also included 240 normozoospermic men as controls. In azoospermic men, we found 18 (7.63\%) gr/gr subdeletions, $11(4.66 \%)$ b1/b3 subdeletions and 2 $(0.85 \%) \mathrm{b} 2 / \mathrm{b} 3$ subdeletions (Table 1$)$. When analysed as per their testicular histology of 181 SCOS patients, $11(6.07 \%$; $P=0.11)$ were found with $\mathrm{gr} / \mathrm{gr}$ type subdeletions, $8(4.41 \% ; P=0.001)$ showed $\mathrm{b} 1 / \mathrm{b} 3$, whereas $2(1.16 \% ; P=0.1)$ showed $\mathrm{b} 2 / \mathrm{b} 3$ subdeletions. Similarly, in 55 patients with SA, 7 were found to have $(12.72 \% ; P=0.001)$

Table 1 Number and type of subdeletions among infertile men and men with normal spermatogenesis

\begin{tabular}{lcccc}
\hline Type & No. of patients & gr/gr Deletion & b1/b3 Deletion & b2/b3 Deletions \\
\hline SCOS & 181 & $11(P=0.11)$ & $8\left(P=0.001^{*}\right)$ & $2(P=0.1)$ \\
SA & 55 & $7\left(P=0.002^{*}\right)$ & $3\left(P=0.0003^{*}\right)$ & - \\
Oligospermia & 182 & $12(P=0.07)$ & $5\left(P=0.0098^{*}\right)$ & $4\left(P=0.02^{*}\right)$ \\
Total & 418 & $30\left(P=0.02^{*}\right)$ & $16\left(P=0.002^{*}\right)$ & $6(P=0.06)$ \\
Controls & 240 & 7 & - & -
\end{tabular}

Abbreviations: SA, spermatogenic arrest; SCOS, sertoli cell-only syndrome. Fisher's exact test (one-sided). ${ }^{*}$ Significant at $P<0.05$. 


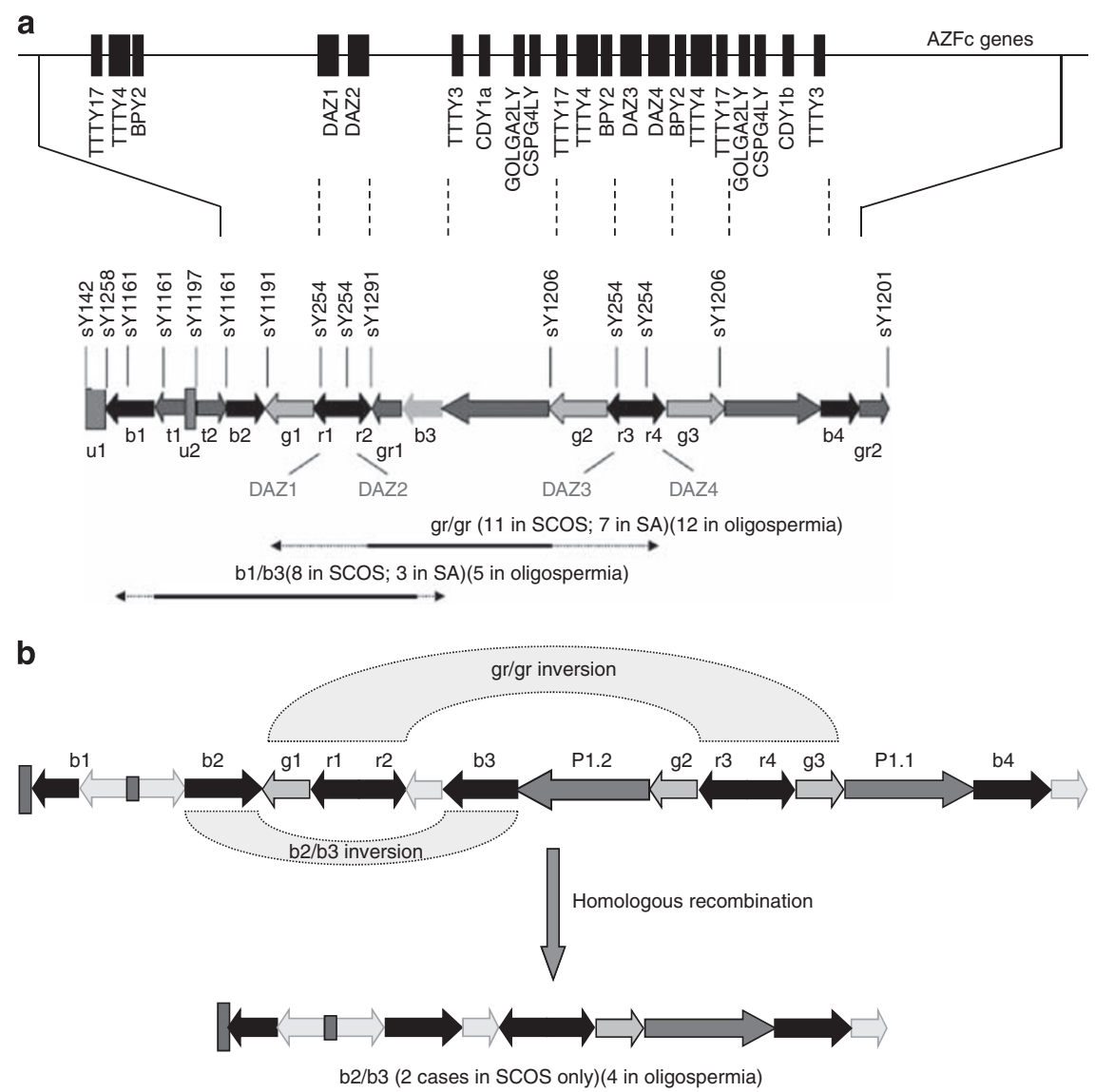

Figure 1 (a) The AZFc region of $Y$ chromosome showing different subdeletions: gr/gr and b1/b3 subdeletions in infertile patients. Repeat sequences are shown with arrows along with the position of various STS markers used in this study. The central bar depicts the organization of the amplicons, including those labeled b1 through b4. It also shows more details about the location of different multicopy genes modified from Skaletsky et al. ${ }^{1}$, (b) b2/b3 subdeletion in infertile patients. Two pathways can lead to this type of deletion. Either gr/gr inversion ( $\mathrm{g} 1, \mathrm{r} 1, \mathrm{r} 2$ recombining with $\mathrm{r} 3, \mathrm{r} 4$ and $\mathrm{g} 3$ ) followed by b2/b3 deletion via homologous recombination or b2/b3 inversion followed by rg/rg deletion via homologous recombination.

gr/gr type subdeletions, $3(5.45 \% ; P=0.0003)$ showed b1/b3 subdeletions. We did not find any $\mathrm{b} 2 / \mathrm{b} 3$ deletions in this group. Similarly, in 182 oligospermic men, we found $12(6.59 \% ; P=0.07) \mathrm{gr} / \mathrm{gr}$ subdeletions, $5(2.74 \% ; P=0.0098) \mathrm{b} 1 / \mathrm{b} 3$ and $4(2.19 \% ; P=0.02) \mathrm{b} 2 / \mathrm{b} 3$ subdeletions. In contrast, screening of 240 normozoospermic men revealed no b1/b3 and b2/b3 subdeletions, whereas 7 (2.91\%) have been found to carry gr/gr subdeletions. Overall, there was a statistically significant difference in the frequency of gr/gr deletions (Fisher's exact test, $P=0.02)$ and b1/b3 $(P=0.002)$ between infertile and fertile men. There was no significant difference in the frequency of b2/b3 subdeletions between infertile and normozoospermic men. If combined, 18 of 236 azoospermic men (7.63\%) had gr/gr subdeletions versus 7 of 240 normozoospermic men (2.9\%; OR: $2.74,95 \%$ CI: $1.13-6.71 ; P=0.02)$, which indicate that men carrying these subdeletions are at significantly higher risk to be azoospermic. Similarly, 12 of 182 oligospermic men (6.59\%; OR: 2.35 , 95\% CI: 0.91-6.09; $P=0.09$ ) have been found with $\mathrm{gr} / \mathrm{gr}$ deletions. Reference examples are shown in Figures 1a, b.

Among normozoospermic men, with gr/gr-deleted genotype, we found significantly lower sperm concentrations (median $32 \times 10^{6} / \mathrm{ml}$ versus $45 \times 10^{6} / \mathrm{ml}, P=0.01$ ), total sperm counts (median $98 \times 10^{6}$ versus $171 \times 10^{6}, P=0.009$ ) and total motile sperm counts (median $53 \times 10^{6}$ versus $67 \times 10^{6}, P=0.06$ ) than normozoospermic men without the deletion (Table 2). Among oligospermic men with gr/gr subdeletions, all parameters stated above were low compared with oligospermic men without gr/gr deletions (Table 2).
Next, we asked the question if $D A Z$ gene copy types have any phenotypic effect on the spermatogenesis. DAZ1/DAZ2 genes are deleted in 11 SCOS cases, whereas DAZ3/DAZ4 genes are deleted in 7 SA cases that also show gr/gr subdeletion. In seven normozoospermic men with gr/gr subdeletions, four have DAZ1/DAZ2 deleted, whereas remaining three have $D A Z 3 / D A Z 4$ genes deleted. In two SCOS cases with b2/b3 deletions, DAZ3/DAZ4 genes were also found to be deleted. The prevalence of gr/gr-DAZ1/DAZ2 deletions in SCOS patients $(11 / 181 ; 6.08 \%)$ was significantly higher compared with normozoospermic men (4/240; $1.6 \%$; OR: 3.81 ; $95 \%$ CI: $1.19-12.201 ; P=0.03)$, in whom as there was no difference with regard to gr/gr-DAZ3/DAZ4 deletion $(3 / 240 ; 1.25 \% ; P=0.26$; data in Supplementary Table 1a). In SA patients, the prevalence of gr/gr-DAZ3/DAZ4 deletions are significantly different (7/55 v 3/240; OR: 11.52 ; $95 \%$ CI: 2.88-46.16; $P=0.0004)$ compared with normozoospermic men.

In oligospermic patients, we found five with $D A Z 1 / D A Z 2$ deletions and seven with $D A Z 3 / D A Z 4$ deletions alongwith gr/gr subdeletions, which are not significantly different compared with normozoospermic men. With the other analysis that helps in further characterization of the types of deletions with either $D A Z$ type or CDY1 missing, we found only two types of deletion pattern: DAZ1/DAZ2+CDY1a and $D A Z 3 / D A Z 4+C D Y 1 b$. In other subdeletion type: $\mathrm{b} 2 / \mathrm{b} 3$, we found two type of missing pattern: DAZ3/DAZ4+CDY1a and DAZ3/DAZ4+ CDY1b. No DAZ gene deletions were found with $\mathrm{SNV}$ analysis in men with normal AZFc-specific STS analysis. When we compared the 
Table 2 Semen analysis among normozoospermic and oligospermic with respect to their gr/gr deletions status

\begin{tabular}{|c|c|c|c|c|}
\hline & $\begin{array}{l}\text { Without gr/gr deletions; } \\
\text { mean } \pm S D N Z(n=233)\end{array}$ & $\begin{array}{l}\text { With gr/gr deletions; } \\
\text { mean } \pm S D N Z(n=7)\end{array}$ & $\begin{array}{l}\text { Without gr/gr deletions; } \\
\text { mean } \pm S D O L(n=170)\end{array}$ & $\begin{array}{l}\text { With gr/gr deletions; } \\
\text { mean } \pm S D O L(n=12\end{array}$ \\
\hline Age (years) & $35 \pm 4.3$ & $32 \pm 2.1$ & $35 \pm 4.6$ & $32 \pm 2.1$ \\
\hline Volume (ml) & $3.6 \pm 1.1$ & $3.1 \pm 0.6$ & $1.8 \pm 1.23$ & $1.7 \pm 1.53$ \\
\hline Concentration $\left(10^{6} / \mathrm{ml}\right)$ & $45(29-73)^{\S}$ & $32(26-51)^{\dagger, \S}$ & $13(7-23)^{\S}$ & $11(5-16)^{\S}$ \\
\hline Morphology (\% normal) & $72(59-81)^{\S}$ & $61(49-74)^{\dagger, \S}$ & $41(26-54)^{\S}$ & $37(22-42)^{\S}$ \\
\hline
\end{tabular}

Abbreviations: NZ, Normozoospermic; OL, oligospermic fertile men.

Significant at $P<0.01$.

Significant at $P=0.009$.

\$Median (range).

${ }^{\text {aAt }}$ least two samples on separate days were analysed.

copy number of $D A Z$ and $C D Y 1$ genes with the type of subdeletion and the semen parameters, we found that these genes have slight impact on the various semen parameters (data in Supplementary Table 1b). Representative examples of gene copy number are given in Figure 2. This observation indicates that these genes have the potential to affect the semen quantity and quality and hence can impact on spermatogenic process.

The overall distribution of Y HGs in these men and their spermatogenic status are shown in Table 3a. The HGs found in the gr/grdeleted azoospermic men include $\mathrm{C}^{\star}, \mathrm{F}^{*}, \mathrm{~J} 1, \mathrm{O} 3 \mathrm{e}$ and R1a1, however, haplotypes $\mathrm{H} 1 \mathrm{a} 2$ and $\mathrm{H} 1 \mathrm{~b}$ are found in normozoospermic men that show gr/gr subdeletions. In $18 \mathrm{gr} / \mathrm{gr}$-deleted azoospermic men there were five different $\mathrm{Y}$ chromosome lineages, suggesting that the AZFc partial deletion occurred at least five times independently. Among seven azoospermic men with SA (with gr/gr deletions), the $\mathrm{HG} \mathrm{C}^{\star}$ and J1 was encountered four and three times, respectively. Among 11 infertile patients (SCOS) with gr/gr deletions, the $\mathrm{HG} \mathrm{F}^{*}$, J1 and R1a1 was encountered thrice, whereas O3e twice only (Table 3b, Figure 3). Among the seven normozoospermic fertile males with gr/gr deletion, all were found to carry H1 HG. Similarly, out of 189 oligospermic men, 12 showed gr/gr subdeletions, $\mathrm{C}^{\star}, \mathrm{F}^{\star}, \mathrm{O} 3 \mathrm{e}$ and $\mathrm{R} 2 \mathrm{Y}$ HGs were seen (Table $3 b$ ).

\section{DISCUSSION}

In the past, a clear association between classical AZF deletions and spermatogenic failure has been established on the basis of welldesigned cause-effect studies. ${ }^{2,6,33,34}$ In this study, we found an increased prevalence of AZFc partial deletions (11.87\%) in infertile men compared with normozoospermic fertile men, suggesting that such mutations represent a risk factor for male infertility. Among these partial AZFc deletions, there were 30 cases with gr/gr subtype, 16 with $\mathrm{b} 1 / \mathrm{b} 3$ and 6 with $\mathrm{b} 2 / \mathrm{b} 3$. The frequency of the $\mathrm{gr} / \mathrm{gr}$ deletions in 418 infertile men from Indian population (7.17\%) was higher than observed in populations from United States $(2.2 \%)$ and European countries $(5.1 \%)$, respectvely. ${ }^{12,13,18,19}$ This variation may be attributed to the different genetic background of the study subjects in these studies. A recent study comprising of infertile men from East Asian population reported a $10.3 \%$ prevalence of $\mathrm{gr} / \mathrm{gr}$ subdeletion. ${ }^{35}$ Frequency of gr/gr deletions in normozoospermic fertile men was much lower $(2.9 \%)$ compared with infertile men $(7.17 \%)$ as found in our study. This is in line with studies from European and Han-Chinese populations. ${ }^{10,17,36}$ The overall difference in the frequencies of subdeletions between Indian and Chinese populations could be because of the genetic background of the study participants. The prevalence of subdeletions such as b1/b3 and b2/b3 only in the infertile group has reinforced our view that these subdeletions might have some impact on spermatogenesis. Similar observations were made with respect to b2/b3 subdeletions in the Han-Chinese population, in which the frequency was significantly high. ${ }^{36}$ However, other studies have reported very low frequencies of these subdeletions and found no association with infertility and/or spermatogenesis. ${ }^{14,16,19,27,34,37}$ Either genetic background of the population studied or the type of infertility might explain the reasons in the frequencies of the different subdeletions.

There are significant differences in various parameters associated with semen quality in men with gr/gr subdeletions, which further reinforces the point that these mutations are associated with the spermatogenesis impairment. This observation is corroborated by other studies as well. ${ }^{13,14,27}$ To further define the genotype-phenotype relationship, we found the role of partial subdeletions (gr/gr; b1/b3 and b2/b3) be more complicated than previously thought. We did not find any b1/b3 and b2/b3 subdeletions in normozoospermic men that further enhance the critical impact of these subdeletions in spermatogenic failure. However, a previous report did not find any association with spermatogenic failure as both $\mathrm{b} 1 / \mathrm{b} 3$ and $\mathrm{b} 2 / \mathrm{b} 3$ subdeletions were found in infertile and fertile group. ${ }^{16}$ Type of infertility and ethnicity of studied population is the most plausible reason for this observation.

Analysis of $D A Z$ gene copy type indicates different impact in azoospermic patients: SCOS with $D A Z 1 / D A Z 2$ deletions, whereas SA with $D A Z 3 / D A Z 4$ genes missing. Our findings in SA patients imply that gr/gr deletions involving $D A Z 3 / D A Z 4$ genes might be the most important risk factor for the spermatogenic impairment. Environmental factors, genetic modifications and probably the Y- chromosome HGs might have an important role in this process. Although gr/gr subdeletion, which either removes DAZ1/DAZ2 or DAZ3/DAZ4 genes, is found in two normozoospermic men, respectively, but this has been found to be fully compatible with proven fertility. This finding does not support the findings from the previously published report that partial gr/gr deletions along with $D A Z 1 / D A Z 2$ gene deletions are significantly associated with infertility and is a risk factor for spermatogenic damage. ${ }^{12,14,25} \mathrm{We}$ also found that $\mathrm{b} 1 / \mathrm{b} 3$ deletion is associated with $D A Z 1 / D A Z 2$ gene deletions to be a risk factor for spermatogenic damage. This can be explained by the smaller length of recombination substrates and hence, the greater predisposition to the condition. A recent study examined the expression of all four $D A Z$ proteins in human testis and found their expression highly polymorphic. ${ }^{38}$

The distribution of various Y chromosome HGs has further helped us to find out the effect of genetic background in determining incidence of these subdeletions. A total of 52 partial AZFc deletions were found in 418 infertile patients. In this study, we reported the 


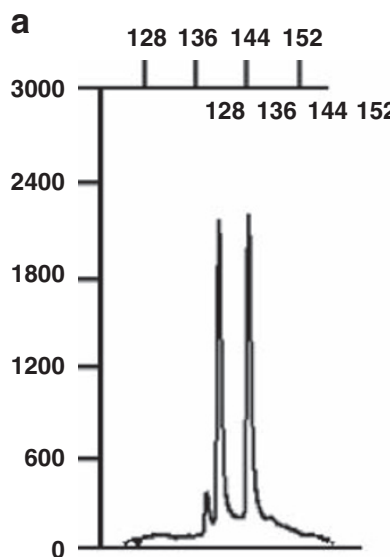

b
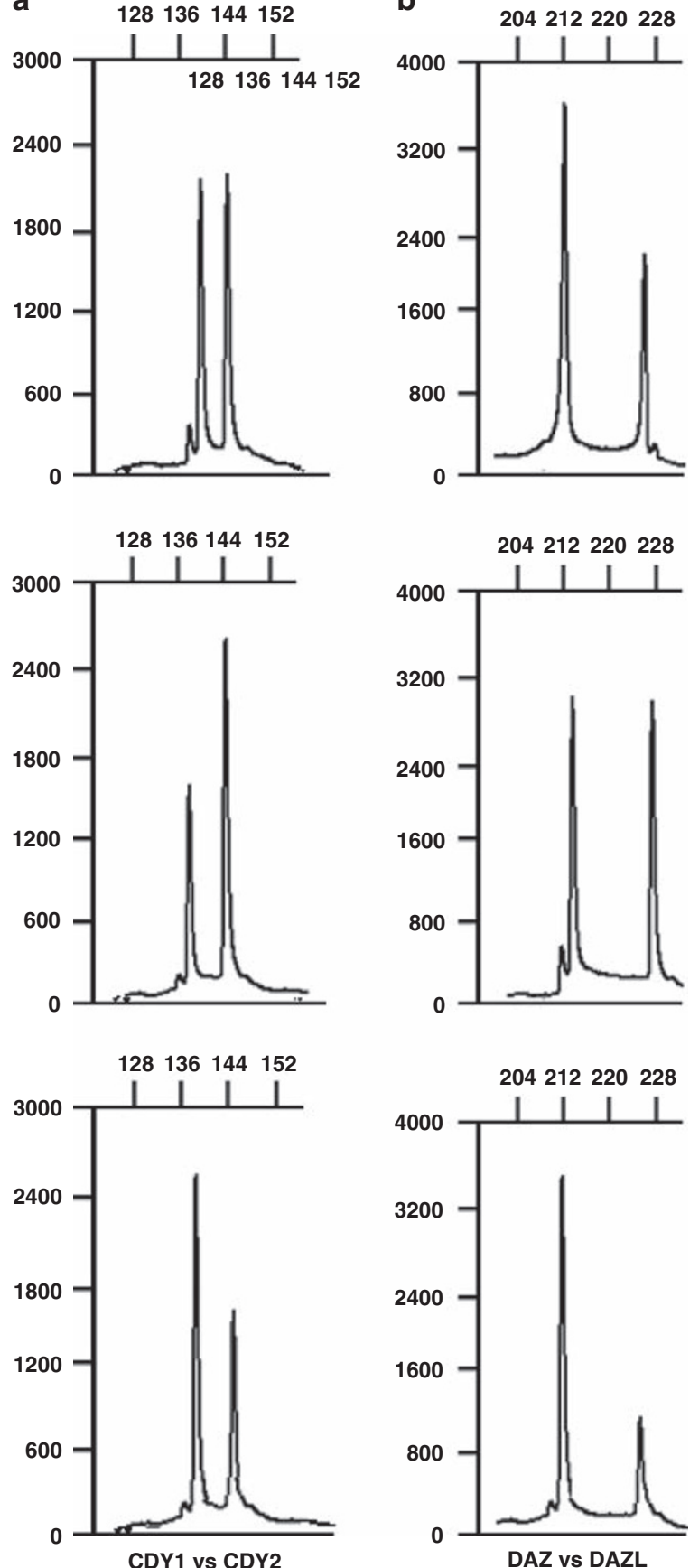
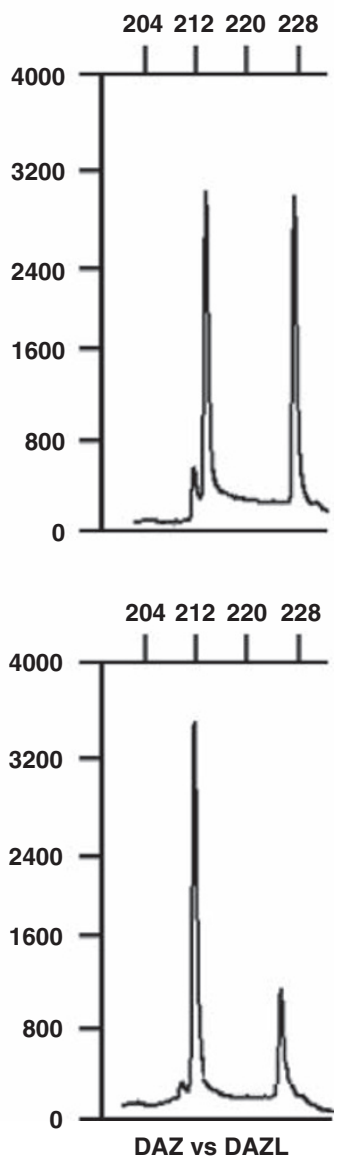

Figure 2 Examples of capillary electrophoretograms showing different gene dosages for the CDY1/CDY2 and DAZ/DAZL genes. The $x$-axis shows length of PCR products (base pairs), whereas $y$-axis shows fluorescent intensity (arbitrary units). The gene dosage of CDY1 and DAZ can be calculated by the comparison of peak area with CDY2 and DAZL, respectively, as the latter acts as internal standard with known number of copies. (a) The peak area of $C D Y 1$ is compared with that of CDY2 (corresponding to two copies). A CDY1/CDY2 pattern of $1: 1$ indicates two CDY1 copies ('normal' CDY1 gene dosage according to the reference sequence; top left), 0.5:1 indicates one $C D Y 1$ copy (middle left) and 1.5:1 indicates three CDY1 copies (bottom left). (b) The peak area of $D A Z$ is compared with that of $D A Z L$ (corresponding to two copies). DAZ/DAZL pattern of 2:1 indicates four $D A Z$ copies ('normal' $D A Z$ gene dosage according to the reference sequence; top right), 1:1 indicates two $D A Z$ copies (middle right) and 3:1 indicates six $D A Z$ copies (bottom right).
Table 3a Overall distribution of $Y$ haplogroups among cases and controls

\begin{tabular}{lrrr}
\hline Haplotype & $\begin{array}{c}\text { Azoospermic } \\
\text { patients }(\mathrm{n}=236)\end{array}$ & $\begin{array}{c}\text { Oligospermic } \\
\text { patients }(\mathrm{n}=182)\end{array}$ & $\begin{array}{r}\text { Fertile men } \\
(\mathrm{n}=240)\end{array}$ \\
\hline C-M216/RPS4Y & 5 & 1 & 2 \\
F-M213/M089 & 12 & 8 & 11 \\
H1a1-M197 & 13 & 9 & 8 \\
H1a2-M097 & 31 & 26 & 43 \\
H1b-M370 & 5 & 3 & 10 \\
J1-M267 & 4 & 4 & 1 \\
J2a-M410 & 10 & 8 & 13 \\
J2b2-M241 & 12 & 12 & 15 \\
L1-M076 & 26 & 13 & 14 \\
O2a-M095 & 33 & 30 & 39 \\
O3e-M134 & 20 & 18 & 19 \\
R1a1-M017 & 36 & 26 & 37 \\
R2-M124 & 29 & 24 & 28 \\
Total & 236 & 182 & 240 \\
\hline
\end{tabular}

Table 3b Proportion of $Y$ haplogroups among cases and controls that show gr/gr, b1/b3 and b2/b3 deletions

\begin{tabular}{llcc}
\hline Spermatogenic status & Haplotype & Infertile men & Fertile men \\
\hline SA & C-M216/RPS4Y & 4 & 0 \\
SCOS & F-M213/M089 & 3 & 0 \\
NZ & H1a2-M097 & 0 & 5 \\
NZ & H1b-M370 & 0 & 2 \\
SA/SCOS & J1-M267 & $3 / 3$ & 0 \\
SCOS & L1-M076 & $8(\mathrm{~b} 1 / \mathrm{b} 3)$ & 0 \\
SCOS & O3e-M134 & 2 & 0 \\
SCOS & R1a1-M017 & 3 & 0 \\
SCOS & R2-M124 & $2(\mathrm{~b} 2 / \mathrm{b} 3)$ & 0 \\
Oligospermic & C-M216/RPS4Y & 1 & 0 \\
Oligospermic & F-M213/M089 & 5 & 0 \\
Oligospermic & J1-M267 & $2(\mathrm{~b} 2 / \mathrm{b} 3)$ & 0 \\
Oligospermic & L1-M076 & $5(\mathrm{~b} 1 / \mathrm{b} 3)$ & 0 \\
Oligospermic & O3e-M134 & 3 & 0 \\
Oligospermic & R1a1-M017 & $2(\mathrm{~b} 2 / \mathrm{b} 3)$ & 0 \\
Oligospermic & R2-M124 & 3 & 0 \\
\hline Abbrevions: NZ, nom & & 0 \\
\hline
\end{tabular}

Abbreviations: NZ, normozoospermic; SA, spermatogenic arrest; SCOS, sertoli cell-only syndrome.

distribution of 13 Y-chromosome HGs in control and patient groups. The results indicate that distribution of three HGs was slightly significantly different in three groups: azoospermic, oligospermic and controls. To further verify the role of population stratification, we also performed HG analysis in 200 individuals with unknown spermatogenic status but with the similar ethnic background. We did not find any significant differences in the distribution of these HGs in this population. We believe that the results obtained in the current case-control study are not influenced by the population stratification. Therefore, we suggest that Y-chromosome background may have a role in the spermatogenic process. Furthermore, we reported four deletion-fixed HGs: the HG F*, R1a1 and R2 (fixation of gr/gr partial deletion) and the HG L1 (fixation of b2/b4 complete deletion). However, we were unable to find any $\mathrm{N} 1$ and Q1 HGs that were found to be associated with b2/b3 deletions to the environmental factors and the genetic background of the study population. ${ }^{39}$ 


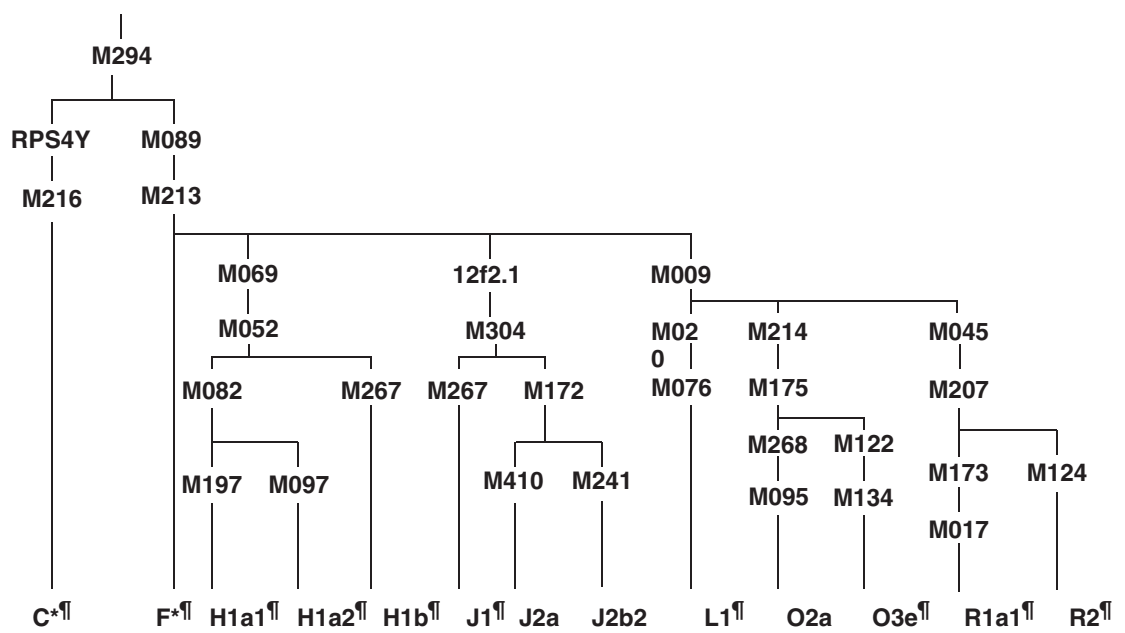

Figure 3 The genealogical tree of human $Y$ chromosome and branches in which gr/gr and b2/b4 deletions were observed in patients and controls. The designation for the branches is based on the $Y$ Chromosome Consortium (2002), which later on is updated by Jobling and Taylor-Smith ${ }^{31}$. *, Indicate that haplogroup may not be monophyletic; $\uparrow$, indicate the haplogroup found in patients and controls with subdeletions, such as gr/gr, b1/b3 and b2/b3.

The AZFc region contains many gene families such as $R B M Y, D A Z$, BPY2, CDY1, PRY, CSPG4LY, GOLGA2LY, TTTY3, TTTY4, TTTY5, TTTY6 and TTTY17 each with multiple copies, with a total of 32 copies. ${ }^{40}$ However, the functional contribution of such genes and transcription units to spermatogenesis is still not fully understood and hence at this stage appears genetically redundant. ${ }^{41}$ All partial AZFc deletions reported so far resulted in the reduction of their copy number, however, a clear dosage effect on sperm production was not seen. They remove 2 copies of the $D A Z$ genes and a variable number of the other genes: the gr/gr deletion subtype removes 1 copy of each of the other genes (a total of 23 gene copies left), and the b1/b3 deletion subtype also removes a copy of BPY2, TTTY4, TTTY5 and TTTY17 and 2 copies of TTTY6 (a total of 20 left), b2/b3 deletion subtype also removes another copy of BPY2, TTTY4 and TTTY17 (a total of 9 gene copies left) and b2/b4 deletions removes 3 copies of $B P Y 2,4$ copies of DAZ and all copies of CSPG4LY, GOLGA2LY and TTTY3 (a total of 11 copies left). As our first screening method is based on STS and SNV analyses, which allow us to define deletions only but not other mutations, such as duplications and inversions, however, other unexpected complex rearrangements may have some role in the spermatogenic process. To answer this question, we used the copy number approach of $D A Z$ and CDY1 genes. ${ }^{16,18}$ When gr/gr subdeletions were first reported in 2003, it was hypothesized that secondary duplication in gr/gr-deleted chromosomes might act as a compensatory mutation by restoring gene copy number. ${ }^{12}$ However, a recent European report showed no such compensatory effect of a secondary duplication after a gr/gr deletion. ${ }^{27}$ This study has shown that gr/gr subdeletions, as such are a significant risk factor as indicated previously by another report. ${ }^{42}$ Therefore, on the basis of present results, it is highly likely that other subdeletions, such as b1/b3 and $\mathrm{b} 2 / \mathrm{b} 3$, should also be considered in the diagnostic screening in case being transmitted to the male offspring. Our normozoospermic men with gr/gr subdeletion were hgrH1a and hgrH1b, whereas no infertile men with either gr/gr, b1/b2 or b2/b3 subdeletions were found to have these HGs. However, in infertile men with b1/b3 subdeletions, HG hgrL1 is found to be deleterious pathogenic. Although these numbers are not large enough, we can not speculate if gr/gr subdeletions are deleteriously pathogenic or $\mathrm{b} 1 / \mathrm{b} 3$ are pathogenic in association with certain HGs.
The results indicate that not only AZFc subdeletions such as gr/gr, b1/b3 and b2/b3 but other specific variations in Y HGs are also associated with impaired spermatogenesis process. Deletions involving $D A Z 1 / D A Z 2+C D Y 1 a$ and DAZ3/DAZ4+CDY1b cluster also appear to represent an actual risk factor for spermatogenic pathology in different phenotype ranging from SCOS to SA to oligospermic phenotype. The inferences from the previous studies indicate that men with partial AZFc deletion(s) may naturally father children. However, if that is not the case, those will opt for assisted reproduction technologies such as in vitro fertilization, which will transmit the mutation to their male children. This study has provided further evidence that these subdeletions act as a risk factor for sperm quality. It is still not clear how and which other factors (genetic and environmental) alter phenotypic effects of these subdeletions. It is therefore concluded that studies of this type will be very useful in providing further information about the genetic causes of male infertility and we believe that the present findings are an important step towards better understanding of the spermatogenesis pathology. But the lack of information so for available about the pathogenic mechanisms underlying the disruption of spermatogenic process suggest the use of these tests in the clinical practice.

\section{CONFLICT OF INTEREST}

The authors declare no conflict of interest.

\section{ACKNOWLEDGEMENTS}

We thank the patients, and other study volunteers for their participation in this study, and the clinician especially to Dr HC Das from the Department of Male Family Welfare Centre, Maulana Azad Medical College and Lok Nayak Jai Prakash and Associated Hospitals, New Delhi, India. This work was supported by funds from the Department of Sciences and Technology to MS to Fast Track Careers of Young Scientists, New Delhi, India (No. SR/FT/L-92/2004).

\footnotetext{
1 Skaletsky H, Kuroda-Kawaguchi T, Minx PJ et al: The male-specific region of the human $Y$ chromosome is a mosaic of discrete sequence classes. Nature 2003; 423: 825-837. 2 Krausz C, Degl'Innocenti S: Y chromosome and male infertility: update, 2006. Front Biosci 2006; 11: 3049-3061.
} 
3 Foresta C, Moro E, Ferlin A: Y chromosome microdeletions and alterations of spermatogenesis. Endocr Rev 2001a; 22: 226-239.

4 Vogt PH, Edelmann A, Kirsch S et al: Human Y chromosome azoospermia factors (AZF) mapped to different subregions in Yq11. Hum Mol Genet 1996; 5: 933-943.

5 Foresta $C$, Moro E, Ferlin A: Prognostic value of $Y$ deletion analysis. The role of current methods. Hum Reprod 2001b; 16: 1543-1547.

6 Simoni M, Bakker E, Krausz C: EAA/EMQN best practice guidelines for molecular diagnosis of y-chromosomal microdeletions. State of the art 2004. Int J Androl 2004; 27: 240-249.

7 Kuroda-Kawaguchi T, Skaletsky $\mathrm{H}$, Brown LG et al: The AZFc region of the $Y$ chromosome features massive palindromes and uniform recurrent deletions in infertile men. Nat Genet 2001; 29: 279-286.

8 Repping S, van Daalen S, Brown $\mathrm{L}$ et al: High mutation rates have driven extensive structural polymorphism among human Y chromosomes. Nat Genet 2006; 38: 463-467.

9 Repping S, van Daalen S, Korver $C$ et al: A family of human $Y$ chromosome has dispersed throughout Northern Eurasia despite a 1.8-Mb deletion in the azoospermia factor c region. Genomics 2004; 83: 1046-1052.

10 Oates RD, Silber S, Brown LG, Page DC: Clinical characterization of 42 oligospermic or azoospermic men with microdeletion of the AZFc region of the $Y$ chromosome, and of 18 children conceived via ICSI. Hum Reprod 2002; 17: 2813-2824.

11 Repping S, Skaletsky H, Lange J et al: Recombination between palindromes P5 and P1 on the human $Y$ chromosome causes massive deletions and spermatogenic failure. Am J Hum Genet 2002; 71: 906-922.

12 Repping S, Skaletsky $\mathrm{H}$, Brown L et al: Polymorphism for a 1.6-Mb deletion of the human $\mathrm{Y}$ chromosome persists through balance between recurrent mutation and haploid selection. Nat Genet 2003; 35: 247-251.

13 de Llanos M, Ballesca JL, Gazquez C, Margarit E, Oliva R: High frequency of gr/gr chromosome $\mathrm{Y}$ deletions in consecutive oligospermic ICSI candidates. Hum Reprod 2005; 20: 216-220.

14 Ferlin A, Tessari A, Ganz F et al: Association of partial AZFc region deletions with spermatogenic impairment and male infertility. J Med Genet 2005; 42: 209-213.

15 Lynch M, Cram DS, Reilly A, O'bryan MK, Baker HW, de Kretser DM, McLachlan RI: The $Y$ chromosome gr/gr subdeletion is associated with male infertility. Mol Hum Reprod 2005; 11: 507-512.

16 Giachini C, Laface I, Guarducci E, Balercia G, Forti G, Krausz C: Partial AZFc deletions and duplications: clinical correlates in the Italian population. Hum Genet 2008; 124: 399-410.

17 Visser $\mathrm{L}$, Westerveld $\mathrm{GH}$, Korver $\mathrm{CM}$ et al: $\mathrm{Y}$ chromosome gr/gr deletions are a risk factor for low semen quality. Hum Reprod 2009; 24: 2667-2673.

18 Machev N, Saut N, Longepied G et al.: Sequence family variant loss from the AZFc interval of the human $\mathrm{Y}$ chromosome; but not gene copy loss; is strongly associated with male infertility. J Med Genet 2004; 41: 814-825.

19 Hucklenbroich K, Gromoll J, Heinrich M, Hohoff C, Nieschlag E, Simoni M: Partial deletions in the AZFc region of the $Y$ chromosome occur in men with impaired as well as normal spermatogenesis. Hum Reprod 2005; 20: 191-197.

20 Ravel C, Chantot-Bastaraud S, El-Houate B, Mandelbaum J, Siffroi J, McElreavey K: GR/GR deletions within the azoospermia factor $c$ region on the $Y$ chromosome might not be associated with spermatogenic failure. Fertil Steril 2006; 85: 229-231.

21 Carvalho CM, Zuccherato LW, Bastos-Rodrigues L, Santos FR, Pena SD: No association found between gr/gr deletions and infertility in Brazilian males. Mol Hum Reprod 2006; 12: 269-273.

22 Kuroki Y, Iwamoto T, Lee J et al: Spermatogenic ability is different among males in different Y chromosome lineage. J Hum Genet 1999; 44: 289-292.
23 Krausz C, Quintana-Murci L, Rajpert-De Meyts E et al: Identification of a Y chromosome haplogroup associated with reduced sperm counts. Hum Mol Genet 2001; 10: 1873-1877.

24 Yang Y, Ma M, Li L et al: Evidence for the association of Y-chromosome haplogroups with susceptibility to spermatogenic failure in a Chinese Han population. $J$ Med Genet 2008; 45: 210-215.

25 Fernandes S, Huellen K, Goncalves J et al: High frequency of DAZ1/DAZ2 gene deletions in patients with severe oligozoospermia. Mol Hum Reprod 2002; 8: 286-298.

26 Giachini C, Guarducci E, Longepied G et al: The gr/gr deletion(s): a new genetic test in male infertility? J Med Genet 2005; 42: 497-502.

27 Krausz C, Giachini C, Xue Y et al: Phenotypic variation within European carriers of the Y-chromosomal gr/gr deletion is independent of Y-chromosomal background. J Med Genet 2009; 46: 21-31.

28 World Health Organization: WHO Laboratory Manual for the Examination of Human Semen and Semen-Cervical Mucus Interactions, . 4th ed. University Press: Cambridge (UK), 1999.

29 Ferlin A, Bettella A, Tessari A, Salata E, Dallapiccola B, Foresta C: Analysis of the DAZ gene family in cryptorchidism and idiopathic male infertility. Fertil Steril 2004; 81: 1013-1018.

30 The Y Chromosome Consortium: A nomenclature for the tree of human Y-chromosomal binary haplogroups. Genome Res 2002; 12: 339-348.

31 Jobling MA, Tyler-Smith C: The human Y chromosome: an evolutionary marker comes of age. Nat Rev Genet 2003; 4: 598-612.

32 Carvalho CM, Pena SD: Optimization of a multiplex minisequencing protocol for population studies and medical genetics. Genet Mol Res 2005; 4: 115-125.

33 Ferlin A, Moro E, Rossi A, Dallapiccola B, Foresta C: The human Y chromosome's azoospermia factor $\mathrm{b}(\mathrm{AZFb})$ region: sequence, structure, and deletion analysis in infertile men. J Med Genet 2003; 40: 18-24.

34 Fernando L, Gromoll J, Weerasooriya TR, Nieschlag E, Simoni M: Y-chromosomal microdeletions and partial deletions of the azoospermia factor $c$ (AZFc) region in normozoospermic, severe oligozoospermic and azoospermic men in Sri Lanka. Asian J Androl 2006; 8: 39-44.

35 Zhang F, Li Z, Wen B et al: A frequent partial AZFC deletion does not Render an increased risk of spermatogenic impairment in East Asians. Ann Hum Genet 2005; 70: 304-313.

$36 \mathrm{Wu}$ B, Lu NX, Xia YK et al: A frequent Y chromosome b2/b3 subdeletion shows strong association with male infertility in Han-Chinese population. Hum Reprod 2007; 22: 1107-1113.

37 Imken L, EI Houate B, Chafik A et al: AZF microdeletions and partial deletions of AZFC region on the $Y$ chromosome in Moroccan men. Asian J Androl 2007; 9: 674-678.

$38 \mathrm{Kim} \mathrm{B}$, Lee Y, Kim Y et al: Polymorphic expression of DAZ proteins in the human testis. Hum Reprod 2009; 24: 1507-1515.

39 Lu C, Zhang J, Li Y et al: The b2/b3 subdeletion shows higher risk of spermatogenic failure and higher frequency of complete AZFc deletion than the gr/gr subdeletion in a Chinese population. Hum Mol Genet 2009; 18: 1122-1130.

40 Yen P: The fragility of fertility. Nat Genet 2001; 29: 243-244.

41 Fernandes S, Paracchini S, Meyer LH, Floridia G, Tyler-Smith C, Vogt PH: A large AZFc deletion removes DAZ3/DAZ4 and nearby genes from men in $\mathrm{Y}$ haplogroup. Am J Hum Genet 2004; 74: 180-187.

42 Tuttelmann F, Rajpert-De Meyts E, Nieschlag E, Simoni M: Gene polymorphisms and male infertility-a meta-analysis and literature review. Reprod Biomed Online 2007; 15: 643-658.

Supplementary Information accompanies the paper on European Journal of Human Genetics website (http://www.nature.com/ejhg) 